\title{
Estimating the probability of a blood donation adverse event based on 1000 interviewed whole-blood donors
}

\author{
B.H. Newman and A.J. Roth
}

\begin{abstract}
BACKGROUND: Estimating the probability that a donor will have or not have an adverse event is useful for staff knowledge to give blood donors reassurance upon request.
\end{abstract}

STUDY DESIGN AND METHODS: One-thousand donors from the general donor pool were interviewed for seven potential adverse events 3 weeks after a $525-\mathrm{mL}$ whole-blood phlebotomy. The four most common adverse events were bruise $(22.7 \%)$, sore arm $(10.0 \%)$, fatigue $(7.8 \%)$, and donor reaction (7.0\%). A stepwise logistic regression analysis was performed based on five donor characteristics that were studied: age, weight, sex, race, and first-time donor status. The contribution of each significant or marginally significant factor to each adverse event was quantified.

RESULTS: For donor reaction, weight $(p<0.0001)$ and age $(p=0.015)$ were significant contributors, and firsttime donor status ( $p=0.054$ ) was a marginally significant contributor. An equation was derived, and the donor reaction rate can be estimated for a group based on the donor's weight, age, and first-time donor status. Similar analyses were performed for fatigue, sore arm, and bruise.

CONCLUSION: Based on the derived formulas and with the use of a spreadsheet, data can be entered and the probability that a donor will have (or not have) a donor reaction, fatigue, sore arm, or bruise can be estimated.

From the American Red Cross Blood Services, Southeastern Michigan Region, and the Wayne State University School of Medicine, Detroit, Michigan; and Pfizer Pharmaceutical Corporation, Ann Arbor, Michigan.

Address reprint requests to: Bruce $\mathrm{H}$. Newman, MD; Medical Director, American Red Cross Blood Services, SE Michigan Region, 100 Mack, Detroit, MI 48201; e-mail: newmanb@usa. redcross.org.

Received for publication November 2, 2004; revision received March 29, 2005, and accepted March 29, 2005.

doi: 10.1111/j.1537-2995.2005.00595.x

TRANSFUSION 2005;45:1715-1721.
S tudies have evaluated the incidence of blood donation-related donor reactions and have also evaluated the incidence of such reactions relative to specific donor characteristics such as age, sex, weight, and first-time donor status. ${ }^{1-5}$ Other adverse events (AEs) have also been evaluated. ${ }^{5,6}$ Most studies were based on observation of donors during the collection process and did not include a postdonation interview. ${ }^{1-4}$ Two recent studies showed that the addition of a postdonation interview increased the detection rate for blood donor AEs from the range of 2.5 to 5.5 percent ${ }^{5}$ to 36 percent. ${ }^{6.7}$ Donor reactions increased from 2.5 to 7.0 percent, ${ }^{6}$ and some AEs, such as bruise, fatigue, and sore arm, are best detected 1 to 3 days after the blood donation. ${ }^{6}$

This study is based on analysis of 1000 randomly selected whole-blood donors who were interviewed for seven AEs 3 weeks after a $525-\mathrm{mL}$ whole-blood donation. ${ }^{6}$ Donors had no AEs, one AE, or more than one AE. The four most common AEs were bruise $(22.7 \%)$, sore arm (10.0\%), fatigue $(7.8 \%)$, and donor reaction $(7.0 \%$-including observation). The other three AEs had incidence rates of less than 2 percent: hematoma (1.7\%), sensory changes in the arm $(0.9 \%)$, and nausea and/or vomiting alone $(0.4 \%)$. We were able to apply logistic regression analysis to donors with and without the four most common AEs to assess which demographic and physical factors (age, weight, sex, race, or first-time donor status) contributed to each of these AEs and to what degree.

\section{MATERIALS AND METHODS}

\section{Blood donor suitability and phlebotomy}

Blood donors met acceptability criteria before being subjected to phlebotomy. The donor then lay in a supine position, and the phlebotomy was performed in the antecubital fossa area of the arm with a 16-gauge needle. The targeted collection volume was $525 \mathrm{~mL}$, which included $33 \mathrm{~mL}$ in blood tubes for postdonation blood tests and $11 \mathrm{~mL}$ in the tubing. Donors were observed for AEs, and reactions and hematomas were recorded on the blood donor records. Donors were also given written advice relative to postdonation complications and the blood center's telephone number to report postdonation events. Ninety-seven percent of the whole-blood dona- 
tions were allogeneic, and 3 percent were autologous or directed whole-blood donations.

\section{Postdonation interview}

Four volunteers interviewed all blood donors from randomly selected blood drives for AEs approximately 3 weeks after the blood donation. Blood donors were asked an open-ended question about systemic symptoms: "Did you have any general symptoms during or after your blood donation?" This was followed with specific questions about three potential events: "Did you have any donor reaction symptoms such as dizziness, sweats, sudden weakness, or unconsciousness?", "Did you develop fatigue after the blood donation?", and "Did you have any nausea or vomiting that was unrelated to a reaction?”. A second open-ended question was then asked about arm problems: "Did you have any arm problems after your blood donation?" This was then followed with specific questions about four potential events: "Did you develop a bruise?", "Did you develop a hematoma?", "Did you develop pain or tenderness at the venipuncture site or elsewhere?", and "Did you develop any sensory changes like numbness, tingling, burning pain, or any other sensation, and where did the sensation(s) occur?". The differences between a bruise and hematoma were explained. By definition, a bruise consists of color changes in skin and was flat, whereas a hematoma consists of a raised collection of blood under the skin. Additional follow-up questions were asked as needed. Answers were documented on the back of the blood donor record.

\section{Data collection}

Blood donor's age, sex, race, self-reported weight, and blood donation status (first-time vs. repeat donor) were recorded from the blood donor's donation record. Race data were collected into a variety of categories but analyzed in only three: Caucasian, African American, and "other." Incidence data for AEs were recorded from both the postdonation interview and the blood donor's donation record.

\section{Statistical analysis}

The original study ${ }^{6}$ was an observation study to determine the incidence of AEs based on observation and a postdonation interview of whole-blood donors. The original intent was to study 250 whole-blood donors, but this was expanded to 1000 donors to increase the probability of finding less common AEs and to improve the study's precision. A study beyond 1000 donor would further increase precision but was not feasible based on labor considerations.

The probability of an AE was represented via a logistic regression model in the form

$$
\mathrm{e}^{\mathrm{R}} /\left(1+\mathrm{e}^{\mathrm{R}}\right),
$$

where $\mathrm{R}$ was potentially dependent on donor age, sex, weight, race, and first-time donation status, as well as interactions between these factors. Stepwise techniques (see Appendix 1) were used to select the factors to be included in the final model for each of the following AEs: donor reaction, fatigue, sore arm, and bruise. A p value was generated to assess each factor's significance for the event. A one-sided test was used when one was able to predict the direction of the potential effect based on previous studies; otherwise, a two-sided test was used. These decisions are noted in Table 2. For each AE, the regression coefficient of each significant or marginally significant factor was used to quantify that factor's contribution to the $R$ value.

\section{RESULTS}

\section{Demographics of the general donor population and interviewed donors}

Table 1 provides a comparison between the general donor population in $2001(\mathrm{n}=211,900)$ and the 1000 donors who were interviewed. The two populations were very similar. Both had a high proportion of Caucasian blood donors, similar proportions of first-time donors, similar proportions of donors under 30 years, and similar median ages. The interviewed population had proportionally more women than the general donor population (55\% vs. $45 \%$ ), possibly because a greater proportion of females can be reached by telephone during the day.

Table 1 also shows the comparisons of donors who were divided into subgroups by sex, donation status (firsttime or repeat), and race (omitting the Other category). Male blood donors weighed $40 \mathrm{lb}$ more on average than female blood donors and had a markedly lower percentage of donors who weighed less than $130 \mathrm{lb}$ (1\% vs. $15 \%$, $\mathrm{p}<0.0001)$. Repeat blood donors were older on average than first-time donors (43 years vs. 22 years), weighed more on average (175 lb vs. $145 \mathrm{lb}$ ), had a lower percentage of donors who weighed less than $130 \mathrm{lb}(6 \%$ vs. $24 \%$, $\mathrm{p}<0.0001)$, and had a lower percentage of females $(53 \%$ vs. $66 \%, \mathrm{p}=0.003)$. African-American blood donors had a higher percentage of women $(74 \%$ vs. $55 \%, p=0.0009)$ and first-time donors ( $25 \%$ vs. $14 \%, \mathrm{p}=0.021$ ) than Caucasian blood donors and weighed slightly more on average (177.5 lb vs. $170 \mathrm{lb})$.

\section{Estimated rates of AEs}

For each AE, Table 2 shows the regression coefficient for the baseline (defined as a 17-year-old Caucasian male repeat donor who weighs $110 \mathrm{lb}$ ), and the regression coefficient for each contributing factor. The sum of the baseline and contributing factor regression coefficients is the 


\begin{tabular}{|c|c|c|c|c|c|c|c|c|c|}
\hline Donor groups & Number & $\begin{array}{c}\text { Women } \\
(\%)\end{array}$ & $\begin{array}{c}\text { First-time } \\
(\%)\end{array}$ & $\begin{array}{c}\text { Caucasian } \\
(\%)\end{array}$ & $\begin{array}{c}\text { African- } \\
\text { American } \\
(\%)\end{array}$ & $\begin{array}{c}\text { Median } \\
\text { age } \\
\text { (years) }\end{array}$ & $\begin{array}{c}<30 \text { years } \\
(\%)\end{array}$ & $\begin{array}{c}\text { Median } \\
\text { weight }(\mathrm{lb})\end{array}$ & $\begin{array}{c}<130 \mathrm{lb} \\
(\%)\end{array}$ \\
\hline 2001 blood center donor population & 211,900 & 45.4 & 19.2 & 89.9 & 6.0 & 41 & 27.2 & * & * \\
\hline Interviewed donors & 1,000 & 55.3 & 16.5 & 86.2 & 6.8 & 41 & 26.9 & 170 & 8.5 \\
\hline \multicolumn{10}{|l|}{ Sex } \\
\hline Men & 447 & NA $†$ & 12.5 & 87.2 & 4.0 & 42 & 23.7 & 190 & 0.7 \\
\hline Women & 553 & NA & 19.7 & 85.4 & 9.0 & 41 & 29.5 & 150 & 14.8 \\
\hline \multicolumn{10}{|l|}{ Experience } \\
\hline Repeat donors & 835 & 53.2 & NA & 88.5 & 6.1 & 43 & 19.4 & 175 & 5.5 \\
\hline First-time donors & 165 & 66.1 & NA & 74.5 & 10.3 & 22 & 64.8 & 145 & 23.6 \\
\hline \multicolumn{10}{|l|}{ Race } \\
\hline African-American & 68 & 73.5 & 25.0 & NA & NA & 39 & 32.4 & 177.5 & 1.5 \\
\hline Caucasian & 862 & 54.8 & 14.3 & NA & NA & 42 & 25.8 & 170 & 8.5 \\
\hline
\end{tabular}

\begin{tabular}{|c|c|c|c|c|}
\hline Contributor & Donor reaction & Fatigue & Sore arm & Bruiset \\
\hline Baseline $^{*}$ & -1.415 & -3.311 & -1.740 & -2.310 \\
\hline Weight & $\begin{array}{l}-0.0162 \times(w t .-110 \mathrm{lb}) \\
p<0.0001(1 \mathrm{~S})\end{array}$ & $\begin{array}{l}\text { Sex-specific (see } \\
\text { weight-by-sex interaction) } \\
p=0.065(1 \mathrm{~S})\end{array}$ & $\begin{array}{l}-0.0058 \times(w t .-110 \mathrm{lb}) \\
p=0.042(1 \mathrm{~S})\end{array}$ & $\begin{array}{l}+0.0048 \times(w t .-110 \mathrm{lb} .) \\
p=0.032(2 S)\end{array}$ \\
\hline Age & $\begin{array}{l}-0.0208 \times(\text { age }-17 \text { years }) \\
p=0.015(1 S)\end{array}$ & NS & $\begin{array}{l}-0.0144 \times(\text { age }-17 \text { years }) \\
p=0.025(1 \mathrm{~S})\end{array}$ & NS \\
\hline Donation status & $\begin{array}{l}+0.499 \text { (if first-time) } \\
p=0.054(1 S)\end{array}$ & $\begin{array}{l}+0.571 \text { (if first-time) } \\
p=0.023(1 \mathrm{~S})\end{array}$ & NS & NS \\
\hline Sex & NS & $\begin{array}{l}+1.626 \text { (if female) } \\
p=0.005(1 S)\end{array}$ & $\begin{array}{l}+0.399 \text { (if female) } \\
p=0.058\end{array}$ & $\begin{array}{l}+1.337 \text { (if female) } \\
p=0.0003(1 S)\end{array}$ \\
\hline Race & NS & NS & $\begin{array}{l}-0.973 \text { (if O) } \\
\mathrm{p}=0.064(2 \mathrm{~S})\end{array}$ & $\begin{array}{l}\text { Sex-specific (see race- } \\
\text { by-sex interaction) } \\
p=0.011 \text { (2S) }\end{array}$ \\
\hline Weight-by-sex interaction & NS & $\begin{array}{l}-0.0131 \times(\text { wt. }-110 \mathrm{lb}) \\
\quad \text { (if female) } \\
p=0.11(2 S)\end{array}$ & NS & NS \\
\hline Race-by-sex interaction & NS & NS & NS & $\begin{array}{l}\dagger(\text { if } A A \text { male) } \\
-0.871 \text { (if AA female) } \ddagger \\
+0.577 \text { (if O male) } \\
-0.503 \text { (if O female) } \ddagger \\
p=0.069 \text { (2S) }\end{array}$ \\
\hline \multicolumn{5}{|c|}{$\begin{array}{l}\text { * For a hypothetical } 17 \text {-year-old Caucasian male repeat donor weighing } 110 \mathrm{lb} . \\
\text { † Tabled values are for all but } A A \text { males, for whom best estimate is essentially } \mathrm{R}=-\infty \text { (probability of bruise }=0 \text { ) } \\
\text { † Not directly comparable to corresponding males without adding in the term for females under "Sex" above } \\
\text { Abbreviations: wt. = weight; } A A=\text { African-American; } O=\text { other race (neither AA nor Caucasian, or donor failed to indicate); NS = not significant; } \\
1 S=\text { p value based on a one-sided test; } 2 S=p \text {-value based on a two-sided test. }\end{array}$} \\
\hline
\end{tabular}

appropriate value of $\mathrm{R}$ to use in the following formula for estimating the probability of the AE based on the contributing factors:

$$
(*) \text { Probability }(A E)=e^{\mathrm{R}} /\left(1+\mathrm{e}^{\mathrm{R}}\right) .
$$

The smaller the $\mathrm{p}$ value for the contributing factor, the stronger the evidence was that the factor contributed to the AE. The following summarizes the contributors for each AE:

- $\quad$ For donor reactions, weight and age were contributors and donation status was a marginal contributor.
- $\quad$ For fatigue, sex and donation status were contributors and weight was a marginal contributor in women (though not in men).

- $\quad$ For sore arm, weight and age were contributors and sex and race were marginal contributors.

- $\quad$ For bruise, sex, weight, and race were contributors. There was also a marginally significant race by sex interaction for bruise, primarily because of the other race category, for which Other men had more bruises and Other women had fewer bruises than their Caucasian counterparts. 


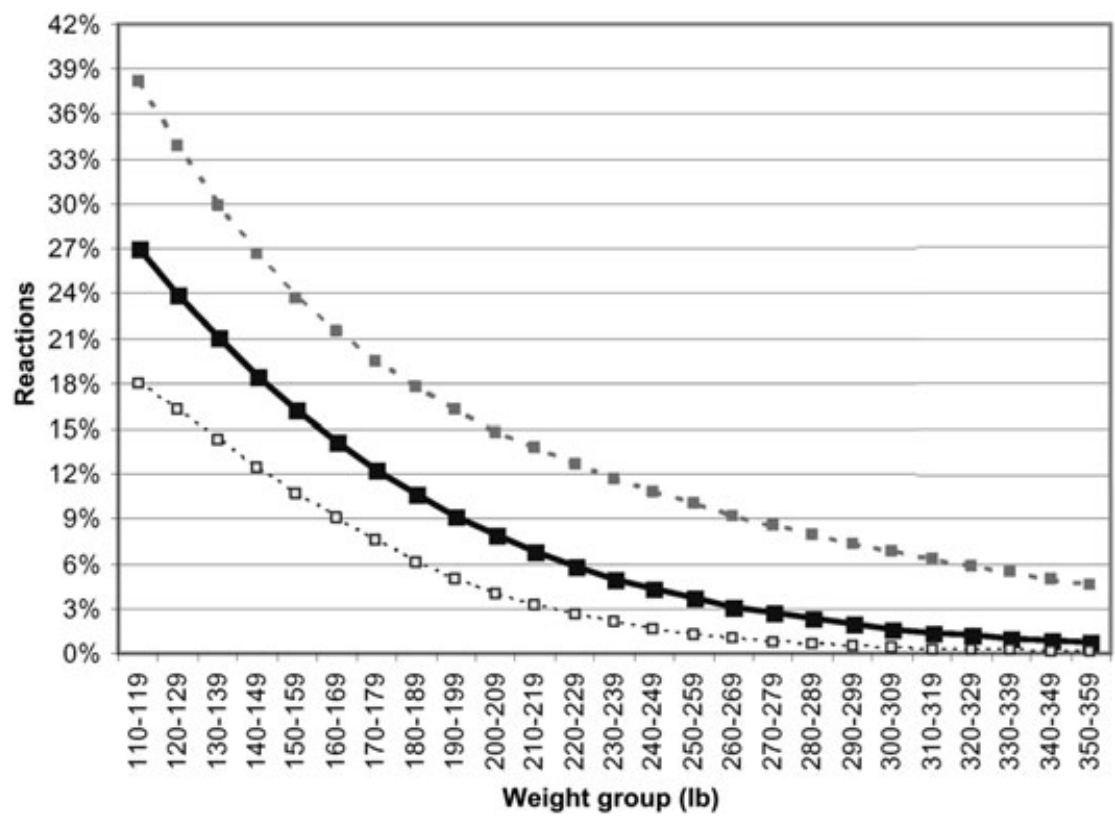

Fig. 1. Estimated donor reaction rates versus weight in first-time, 17-year-old donors (口). (- - $\square$ - -) Upper 95\% CI limit; ( $\square$ ) lower 95\% CI limit.

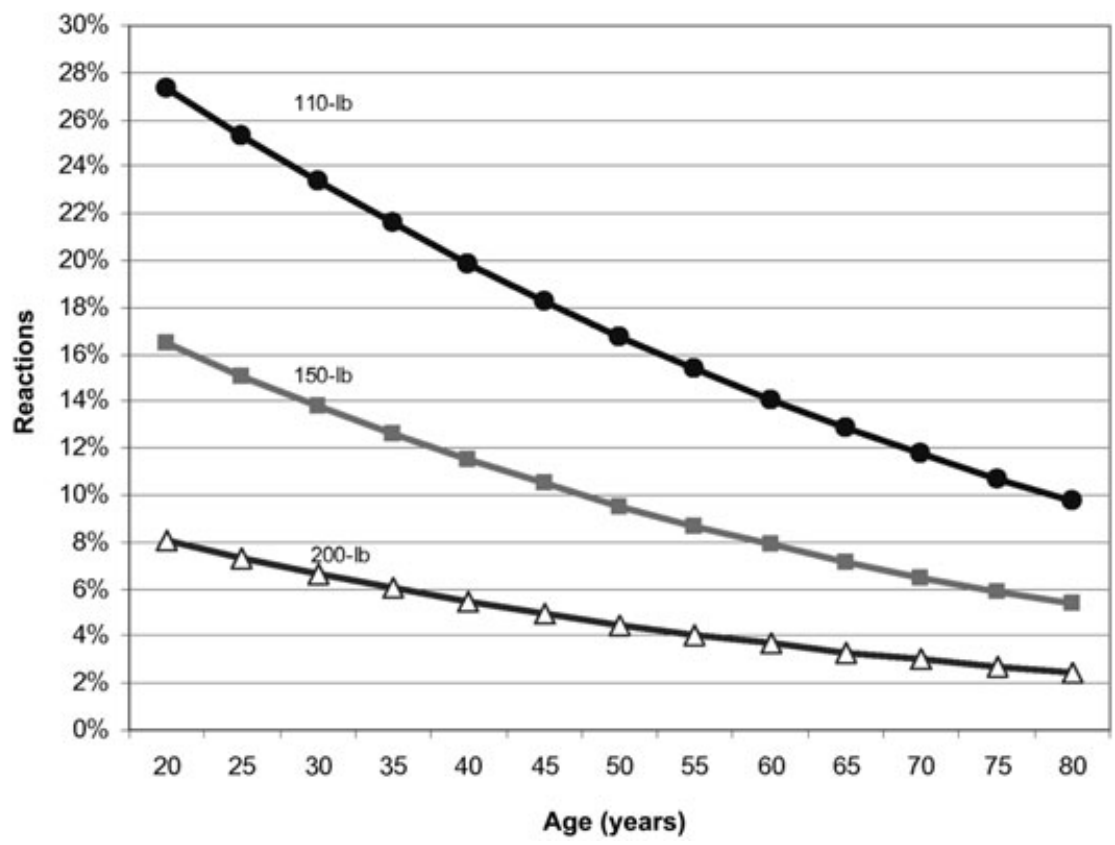

Fig. 2. Estimated donor reaction rates versus age in first-time donors at three different weights. (๑) $110 \mathrm{lb}$; (口) $150 \mathrm{lb}$; $\triangle$ ) $200 \mathrm{lb}$.

\section{Additional descriptions}

Some additional patterns are shown for donor reactions. Figures 1 and 2 are based on repeated applications of the donor reaction rate formula, based on the regression model. They show how donor weight (Figs. 1 and 2) and age (Fig. 2) affect estimated donor reaction rates in first-time donors. It is apparent that donor weight has a very significant impact on donor reactions, and its influence is greater than donor age.

Blood donor weight data are generally not available ${ }^{8}$ because donor weight is generally not entered into a blood center's database. Figure 3 provides weight data for blood donors based on a research study involving 7973 general donors in 1996 and 1997. Figure 3 shows that donor weight increases in men and women with increasing age up to the age of 60 years and then decreases slightly. Changes in donor weight versus donor age help to explain donor reaction rate results from two older studies (see Discussion). ${ }^{3,9}$

\section{DISCUSSION}

A more accurate picture of the blood donation experience can be obtained with the addition of an interview. ${ }^{6,7}$ This study, which is based on interviews with 1000 whole-blood donors, provides a fairly accurate picture of the blood donation experience.

\section{Donor reaction rates}

Weight and age were the most significant contributors to donor reaction rates, and donation status was only marginally significant. Donor weight and age have fairly equivalent regression coefficients ( 0.0162 vs. 0.0208$)$ and therefore influence donor reaction rates to similar degrees per unit increase. Weight, however, can be increased to a greater extent (vs. $110 \mathrm{lb}$ ) than age (vs. 17 years old), so weight is a more important factor in determining donor reaction incidence rates.

One can estimate the probability of a donor reaction based on the donor's weight, age, and first-time-donor status; and this calculation can be easily accomplished in a computer spreadsheet. In the worst-case (and least precise) scenario, where the donor weighs $110 \mathrm{lb}$, is 17 years old, and is a first-time donor, the estimated probability for a donor reaction is highest, at 28.6 percent (with a $95 \%$ confidence interval [CI] of $18.9 \%-40.7 \%$ ) If the same 17 -year- 


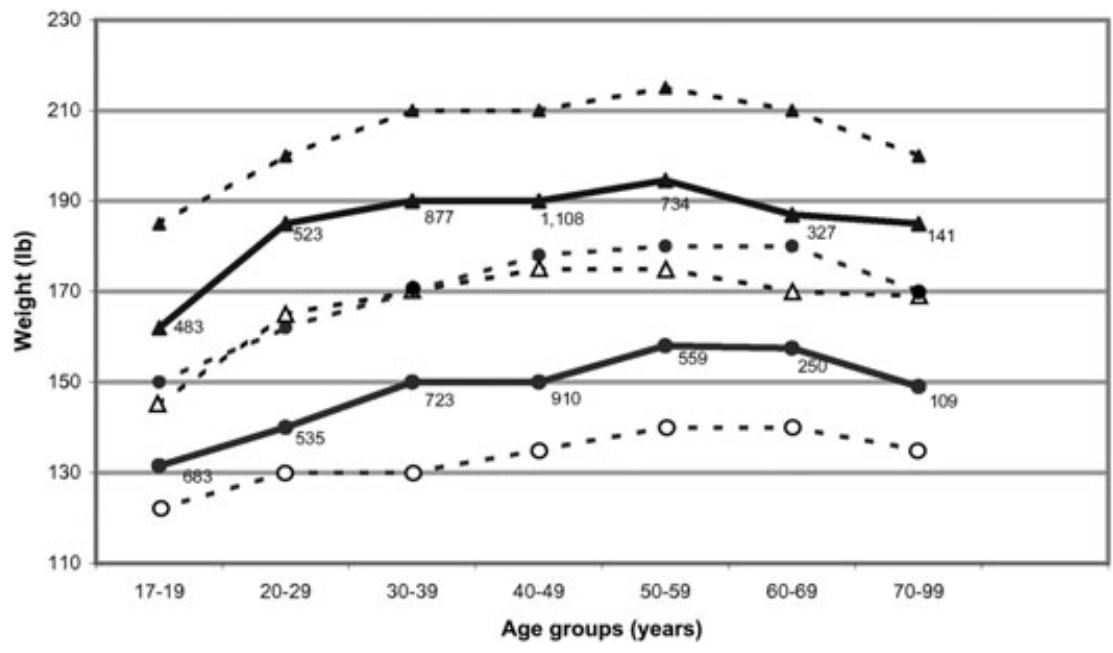

Fig. 3. Male and female donor weight versus age. (-A-) Male median; $(\triangle)$ male 25\%; (- - $\Delta$ - -) male 75\%. (-๑-) Female median; $(\bigcirc)$ female 25\%; (- -๑- -) female $75 \%$. have a higher incidence of iron deficiency. ${ }^{11,12}$ Therefore, it is possible that some or all of the following might contribute to the higher percentage of fatigue in women: greater relative blood volume loss, greater relative $\mathrm{Hb}$ loss, and greater probability of being iron deficient and unable to replace the lost $\mathrm{Hb}$.

The estimated probability of fatigue is 24.7 percent ( $95 \% \mathrm{CI}, 15.8 \%-36.5 \%$ ) in the worst-case scenario where the donor is female, weighs $110 \mathrm{lb}$, and is a first-time donor. If the same woman weighs $250 \mathrm{lb}$, the estimated fatigue rate is just 5.0 percent (95\% CI, 1.5\%-15.3\%). In contrast, a repeat male donor of any weight has an estimated fatigue rate of 3.5 percent (95\% CI, 1.1\%-10.7\%). old, first-time donor weighs $250 \mathrm{lb}$, however, the estimated probability is just 4.0 percent (95\% CI, 1.0\%-10.4\%), which shows that increasing weight is quite protective, even if the donor has other high-risk factors.

The study by Tomasulo and colleagues ${ }^{3}$ in 1980 showed that donor reaction rates in first-time donors between ages 40 to 49 years and over age 50 years were two-thirds less than in first-time donors under the age of 30 years. The study by McVay and coworkers ${ }^{9}$ in 1990 also showed that first-time allogeneic donors between the ages of 30 to 65 years had a 53 percent decrease in donor reactions in comparison to first-time allogeneic donors between the ages of 17 and 29 years. In both studies, increased age alone cannot account for the dramatic fall in donor reaction rates, but the combination of increased age and the accompanying increased weight with age (Fig. 3) does account well for the drop in the donor reaction rate in both studies.

\section{Fatigue rate}

Being female was the most significant contributor to developing fatigue after the blood donation $(\mathrm{R}=+1.626)$. Being a first-time donor also contributed but to a much lesser degree $(\mathrm{R}=+0.571)$. Fatigue in women was mitigated with increasing donor weight. The fact that fatigue occurred more commonly in women than men and that it was mitigated by increasing weight in women (but not men) suggests that size alone is not the sole cause of the fatigue. Women on average have lower total body hemoglobin $(\mathrm{Hb})$ content than men because of smaller blood volumes and lower $\mathrm{Hb}$ concentrations. ${ }^{10}$ The whole-blood collection volume is fixed at a set volume $(525 \mathrm{~mL})$, and therefore a higher proportion of blood volume and $\mathrm{Hb}$ is removed from women than men. In addition, women

\section{Sore arm rate}

Donor weight and age were the most important contributors to the sore arm rate, whereas being female was marginally associated with the sore arm rate. The estimated sore arm rate is 20.7 percent $(95 \% \mathrm{CI}, 14.3 \%-29.1 \%)$ in the worst-case scenario, where the donor is female, weighs $110 \mathrm{lb}$, and is 17 years old. The estimated sore arm rate decreases to 11.4 percent (95\% CI, $8.5 \%-15.1 \%$ ), if the same donor weighs $150 \mathrm{lb}$ and is 50 years old and 6.7 percent (95\% CI, 3.5\%-12.6\%), if the same donor weighs $250 \mathrm{lb}$ and is 50 years old. In contrast, a heavy, older male donor who weighs $250 \mathrm{lb}$ and is 60 years old has an estimated sore arm rate of just 4.0 percent $(95 \%$ CI, $2.2 \%-7.1 \%)$.

\section{Bruise rate}

Women are very susceptible to bruises in comparison to men. ${ }^{6}$ African-American donors are less susceptible to bruises than Caucasian donors, particularly AfricanAmerican men, although the data are limited to just 18 African-American men. Increasing weight is associated with a slight increase in bruise rates.

The estimated bruise rate is 42.5 percent $(95 \% \mathrm{CI}$, $32.2 \%-53.6 \%$ ) in an at-risk scenario where the donor is female and Caucasian and weighs $250 \mathrm{lb}$; but if the same donor weighs $130 \mathrm{lb}$, the estimated bruise rate decreases to 29.4 percent (95\% CI, $24.8 \%-34.3 \%$ ). In contrast, a 175 lb Caucasian man would have an estimated bruise rate of 11.9 percent (95\% CI, 9.0\%-15.6\%).

In conclusion, with stepwise logistic regression analysis techniques on five potential contributing factors (age, weight, sex, race, and first-time donor status), we 
determined which factors contributed and to what degree for common AEs (donor reaction, fatigue, sore arm, bruise). This is useful for staff to provide reassurance to donors upon request and to help identify high-risk groups for preventative studies. A better understanding of contributors could also lead to better preventative strategies.

\section{REFERENCES}

1. Trouern-Trend JJ, Cable RG, Badon SJ, Newman BH, Popovsky MA. A case-controlled multicenter study of vasovagal reactions in blood donors: influence of sex, age, donation status, weight, blood pressure, and pulse. Transfusion 1999;39:316-20.

2. Kasprisin DO, Glynn SH, Taylor Miller KA. Moderate and severe reactions in blood donors. Transfusion 1992;32:23-6.

3. Tomasulo P, Anderson AJ, Paluso MB, Gutschenritter MA, Aster RH. A study of criteria for blood donor deferral. Transfusion 1980;20:511-8.

4. Newman BH. Vasovagal reactions in high school students: findings relative to race, risk factor synergism, female sex, and non-high school participants. Transfusion 2002;42: 1557-60.

5. Newman BH. Donor reactions and injuries from wholeblood donation. Transfus Med Rev 1997;11:64-75.

6. Newman BH, Pichette S, Pichette D, Dzaka E. Adverse effects in blood donors after whole-blood donation: a study of 1,000 blood donors interviewed 3 weeks after whole-blood donation. Transfusion 2003;43:598-603.

7. Sojka P, Sojka BN. Adverse effects in blood donors after whole blood donation: you find what you look for! [letter]. Transfusion 2004;44:135-6; author reply 136.

8. Proceedings of Blood Products Advisory Committee [monograph on the Internet]. Gaithersburg (MD): Food and Drug Administration, Blood Products Advisory Committee; 2003 Dec 12 [cited 2005 Aug. 7]. p. 104. Available from: http://www.fda.gov/ohrms/dockets/AC/03/transcripts/ 4014T2.doc

9. McVay PA, Andrews A, Kaplan EB, et al. Donation reactions among autologous donors. Transfusion 1990;30:249-52.

10. Rushton DH, Dover R, Sainsbury W, et al. Why should women have lower reference limits for haemoglobin and ferritin concentrations than men? Br Med J 2001;322:1355-7.

11. Looker AC, Dallman PR, Carroll MD, Guner EW, Johnson CL. Prevalence of iron deficiency in the United States. JAMA 1997;277:973-6.

12. Iron deficiency-United States, 1999-2000. MMWR Morb Mortal Wkly Rep 2002;51:897-9.

\section{APPENDIX 1: STATISTICS}

It is important to note that the $\mathrm{AE}$ formulas, which are collectively explained and denoted by an asterisk in the section "Estimated rates of AEs," contain several potential sources of uncertainty. For example, we have included marginally significant terms in the model for each AE; by definition, the regression coefficients for these terms are not quite significantly different from zero; that is, there is somewhat more than a 5 percent chance that they are not contributors at all. Although inclusion of marginally significant contributors enhances the accuracy of the estimates, this is obviously one source of substantial uncertainty for at least some estimates.

The uncertainty in the estimates can be quantified. For each AE, and for any given set of values for the contributors, the statistical model can produce a standard error for R, from which we can obtain a CI for R that can be transformed into a CI for the estimated probability

$$
\mathrm{e}^{\mathrm{R}} /\left(1+\mathrm{e}^{\mathrm{R}}\right) .
$$

A narrow $\mathrm{CI}$ indicates that the corresponding probability has been estimated precisely, whereas a wide CI indicates an imprecise estimate with a lot of uncertainty.

It turns out that most of the probabilities are estimated with more than enough precision for practical use, but a few of them have very wide CIs and should be regarded as just very general "ballpark" estimates. In particular, in any regression model, estimates near the "middle" of the data are more precise than estimates extrapolated to (or beyond) the extremes of the data; thus, in our application, estimates that depend on weight or age are fairly precise near the middle of the observed weight and age ranges but far less precise for extremely heavy, light, old, or young donors. Also, estimated probabilities are almost always most variable when they are near 50 percent probability and gain precision as they move toward the extremes of 0 and 100 percent probability. For these two reasons, our least precise estimates are usually the worst-case scenarios, in which the donor usually has extremely low (or extremely high) weight and extremely low age and in which the donor usually has a much higher (and much closer to 50\%) estimated probability of AE than the average donor. In some of the worst-case (and hence least precise) scenarios, the 95 percent CIs are provided under Discussion to give the reader a feel for the amount of variability typically inherent in such estimates.

The statistical analyses were carried out with SAS. A preliminary regression model was selected with the STEPWISE option in PROC LOGISTIC. For reasons described below, this preliminary model was chosen to be quite a bit broader (i.e., containing more contributing factors) than the final model, and this was accomplished via liberal values for the entry criterion $($ SLENTRY $=0.3$ ) and the removal criterion $(S L S T A Y=0.35)$. In approximate terms that are not statistically rigorous, the preliminary model retained all contributing factors whose $\mathrm{p}$ values were below 0.3 . The resulting preliminary model was checked for goodness of fit with the Hosmer and Lemeshow test obtained with the LACKFIT option on the MODEL state- 
ment; there was no significant lack of fit for the chosen model for any of the AEs.

In general, only the significant and near-significant $(p<0.10)$ terms in the preliminary model were retained in the final model. Some subjective judgment (illustrated below) was also used, however, and the above-mentioned liberal criteria ( 0.30 and 0.35 ) for inclusion in the preliminary model were specifically aimed at retaining some nonsignificant terms to which such judgment could be applied. For example, Table 2 indicates a weight-by-sex interaction term for fatigue even though $p=0.11$. This interaction term was included because 1) it induced more intuitively sensible estimates of the regression coefficients for sex and weight individually, 2) the regression coefficient for the interaction was large enough to have a substantial influence on the final estimate in some cases, 3) the $\mathrm{p}$ value was based on a two-sided test in a situation where the above argument based on $\mathrm{Hb}$ provides a plausible (but post hoc) argument for a one-sided test that would have cut the p value in half, 4) the final model seemed to fit the data better with this term than without it, and 5) the $p$ value was only slightly above 0.10 anyway. It would be prudent to rely only on a very experienced practitioner of statistics to make this sort of subjective judgment.

Once the final model was chosen with the above (objective and subjective) considerations, the final regression coefficients (and their associated p values) were obtained with PROC GENMOD for highly technical reasons, but we have no strong objections to the continued use of PROC LOGISTIC for this part of the analysis as well. The 95 percent CIs displayed under Discussion for certain specific cases were obtained via ESTIMATE statements within PROC GENMOD. 